\title{
Criminologie
}

\section{Délinquance juvénile et société}

\section{Alice Parizeau}

Volume 8, numéro 1-2, 1975

Délinquance juvénile au Québec

URI : https://id.erudit.org/iderudit/017046ar

DOI : https://doi.org/10.7202/017046ar

Aller au sommaire du numéro

Éditeur(s)

Les Presses de l'Université de Montréal

ISSN

0316-0041 (imprimé)

1492-1367 (numérique)

Découvrir la revue

Citer ce compte rendu

Parizeau, A. (1975). Compte rendu de [Délinquance juvénile et société].

Criminologie, 8(1-2), 189-194. https://doi.org/10.7202/017046ar d'utilisation que vous pouvez consulter en ligne.

https://apropos.erudit.org/fr/usagers/politique-dutilisation/ 
DÉLINQUANCE JUVÉNILE ET SOCIÉTÉ Note de lecture

Alice Parizeau 
Peut-on mesurer l'importance du phénomène d'inadaptation des jeunes aux normes imposées dans une société donnée?

Il est excessivement difficile de répondre à cette question. Ce qu'on sait c'est que le nombre des mineurs, qui comparaissent devant les juges, augmente, mais il est impossible de préciser si cela est dû surtout à la diminution de l'importance de l'autorité parentale et scolaire, ou plutôt à la remise en question des valeurs proposées par la collectivité aux adolescents et rejetées par eux avec une violence inconnue auparavant.

A cet égard, les recherches comparatives, faites dans des sociétés dont la culture et le stade du développement économique diffèrent, sont peut-être plus révélatrices. Elles permettent de dégager, en effet, certaines constantes qui représentent des indicateurs globaux de l'évolution des comportements des jeunes. On peut, à partir de là, mieux comprendre et mieux cerner des réalités, ce qui est d'autant plus valable que toutes les politiques préventives en dépendent.

L'ouvrage de Hanna Malewska et de Vincent Peyre ', constitue une tentative intéressante dans ce sens, tout en suscitant, à travers ses conclusions, une reflexion sur l'avenir des sociétés post-industrielles.

$\mathrm{Au}$ départ, pour situer le problème, il y a à la base les deux dimensions statistiques; polonaise et française. Entre 1955 et

1. Délinquance juvénile, famille et société, par Hanna Malewska et Vincent Peyre, Centre de formation et de recherche de l'éducation surveillée; Vaucresson, France, 1974. 
1964, le nombre de mineurs jugés par les tribunaux des enfants a passé de 14000 à 42500 en France, tandis qu'en Pologne, pour le seul groupe d'âge de 13 à 16 ans, il a pratiquement doublé, en passant de 11400 à 21000 . Dans les deux pays, dont les structures socio-politiques sont différentes et dont les traditions historiques, culturelles et pédagogiques impliquent la transmission des valeurs dont l'ordre d'importance n'est pas similaire, la délinquance juvénile connue des cours s'accroît plus vite que la popullation. Il s'agit là, il va sans dire, d'un processus qu'il est urgent d'analyser afin de comprendre ses causes et d'élaborer en conséquence des politiques préventives se situant dans trois cadres : familial, scolaire et celui de l'action de l'ensemble de la collectivité.

En ce qui a trait au cadre familial, on sait qu'il est le principal responsable des comportements marginaux des enfants. En dehors cependant des parents incapables, pour diverses raisons socio-économiques, de remplir leurs obligations, se situent tous ceux qui échouent dans leur rôle d'éducateurs sans qu'on puisse uniformiser la justification de cet état de choses. Comme seule constante, on peut considérer l'intensité de la relation parentsenfants, autant en Pologne qu'en France, et cela bien que dans les deux pays l'échelle des punitions et des récompenses est perçue de façon fort différente. Il semble, en somme, qu'en dernier ressort c'est cette relation, en ce qui a trait à son aspect affectif, qui demeure le facteur prépondérant de la socialisation harmonieuse de l'enfant.

En ce qui concerne l'intégration dans le milieu scolaire, elle dépend pour une large part de la qualité de l'éducation familiale, mais cela n'est pas nécessairement vrai dans le domaine de la capacité d'absorption des connaissances. L'accroissement du nombre d'élèves et de la charge qu'il impose aux enseignants, a diminué en outre l'importance de l'encadrement scolaire qui se substituait, ou qui complétait, celui de la famille. Tout se passe comme si l'école d'aujourd'hui transmettait des connaissances, mais ne pouvait, ou ne voulait plus, inculquer certaines valeurs morales et nationales, comme ce fut le cas par le passé.

À la suite de l'éclatement de protection des deux cadres traditionnels, celui de la famille et de l'école, c'est donc la société, dans le sens global de ce terme, qui a une action directe sur l'évolution des jeunes. On peut mentionner à cet égard tout aussi bien 
la qualité de l'environnement, que celle des loisirs municipaux, ou régionaux, ainsi que certains facteurs spécifiques d'identification, dont le modèle de réussite sociale proposé par les media d'information, et la propension à consommer qui impose fatalement de multiples tensions.

C'est ainsi que les auteurs de l'ouvrage Délinquance juvénile, famille, école et société ont pu établir la relation entre le développement socio-économique, analysé dans les deux pays, la France et la Pologne, et la délinquance juvénile telle que définie dans les codes. Ils ont pu démontrer aussi que la délinquance juvénile est systématiquement plus importante dans les régions fortement industrialisées et urbanisées. Les écarts entre ces régions et celles moins développées sont de simple au double, ou même triple. La typologie des délits est, par contre, relativement similaire, puisque dans les deux pays ce sont les délits contre les biens qui prédominent, tandis que le volume de la délinquance contre les personnes et les mours demeure variable.

Parallèlement, les auteurs soulignent certaines contradictions. Ils indiquent, par exemple, qu'en général, la proportion des jeunes jugés comme délinquants est plus élevée parmi ceux qui quittent précocement leurs études, mais dans les régions plus développées, les jeunes restent en moyenne plus longtemps à l'école et cela n'empêche pas le fait que le taux de délinquance y est systématiquement plus élevé. Dans le même ordre d'idées, bien que la délinquance juvénile soit plus répandue dans les familles économiquement faibles, nombreuses, ou désunies, dans les régions plus développées, où les revenus familiaux sont plus. élevés, cela ne semble pas empêcher l'accroissement quantitatif des jeunes connus et jugés par les cours pour mineurs.

Fait significatif, des recherches faites au Québec, dans une perspective régionale, ont permis d'obtenir des résultats sensiblement similaires, bien que le contexte socio-économique de base y est différent de celui qui prévaut en France et en Pologne.

Doit-on conclure dès lors avec Hanna Malewska et Vincent Peyre que si «le développement», n'aide pas ceux pour qui il n'est guère plus, actuellement, qu'un mirage, c'est que les perspectives de ce développement, les moyens employés, les rapports sociaux qui lui correspondent, ne répondent pas pleinement, il s'en faut, à ce qu'on doit en attendre? > 
Dans ce sens, le livre Délinquance juvénile, famille, école et société est, à notre avis, un ouvrage important. Il rompt, en effet, avec la traditionnelle approche selon laquelle la cellule familiale peut et doit assumer tout le poids de la responsabilité de la socialisation des mineurs, en insistant sur la responsabilité collective. Une responsabilité, que les sociétés assument dans le domaine de la punition, mais qu'elles ne semblent pas être prêtes à envisager de façon globale en ce qui a trait à la prévention. En d'autres termes, les sociétés se protègent contre les dangers éventuels de victimisation, mais ne semblent pas vouloir évaluer à priori le prix du développement économique et industriel en ce qui concerne leurs effets négatifs.

Cela signifie que, dans l'immédiat, la grande masse des jeunes est de plus en plus exposée, dans les sociétés les plus industrialisées, aux déséquilibres socio-affectifs qui se traduisent par un accroissement constant des actes et des comportements marginaux.

C'est là une des conclusions des recherches effectuées par H. Malewska et V. Peyre, dont les multiples implications devraient être prises en considération, à notre avis, autant par les chercheurs que par les autorités publiques responsables des politiques préventives d'aujourd'hui et de demain. 